Research Paper

\title{
Involvement of Aryl Hydrocarbon Receptor and Aryl Hydrocarbon Receptor Repressor in Helicobacter Pylori-related Gastric Pathogenesis
}

Renfei Zhu ${ }^{1,2^{*}}$, Cheng Gao ${ }^{1^{*}}$, Liuhua Wang ${ }^{*}$, Guoxin Zhang 3 , Weiming Zhang4, Zhihong Zhang4, Lizong Shen ${ }^{1 凶}$, Shoulin Wang ${ }^{5^{\bowtie}}$

1. Division of Gastrointestinal Surgery, Department of General Surgery, First Affiliated Hospital, Nanjing Medical University, Nanjing 210029, China

2. Department of Hepatobiliary Surgery, Third People's Hospital of Nantong, Nantong 226000, China

3. Department of Gastroenterology, First Affiliated Hospital, Nanjing Medical University, Nanjing 210029, China

4. Department of Pathology, First Affiliated Hospital, Nanjing Medical University, Nanjing 210029, China

5. School of Public Health, Nanjing Medical University, Nanjing 211166, China

"These authors contributed equally to this work.

$\triangle$ Corresponding authors: Prof. L. Shen, Division of Gastrointestinal Surgery, Department of General Surgery, First Affiliated Hospital, Nanjing Medical University, 300 Guangzhou Road, Nanjing, Jiangsu 210029, China; Tel/Fax: +86-25-83724440; E-mail: shenlz@njmu.edu.cn and Prof. S. Wang, Department of Occupational Medicine and Environmental Health, School of Public Health, Nanjing Medical University, 101 Longmian Avenue, Nanjing, Jiangsu 211166, China; Tel/Fax: +86-25-86868417; E-mail: wangshl@njmu.edu.cn

(C) Ivyspring International Publisher. This is an open access article distributed under the terms of the Creative Commons Attribution (CC BY-NC) license (https://creativecommons.org/licenses/by-nc/4.0/). See http://ivyspring.com/terms for full terms and conditions.

Received: 2018.03.15; Accepted: 2018.06.09; Published: 2018.07.01

\begin{abstract}
Background: Persistent Helicobacter pylori $(H$. pylori) infection leads to various gastric diseases. Multiple studies have demonstrated that aryl hydrocarbon receptor (AHR) plays roles in the antibacterial response and aryl hydrocarbon receptor repressor (AHRR) is downregulated in stomach cancer. However, the role of AHR or AHRR in $H$. pylori-related gastric diseases remains unclear.

Aims: To investigate whether AHR or AHRR is involved in $H$. pylori-related gastric diseases.

Methods: Patients with gastritis or gastric adenocarcinoma were enrolled randomly, and gastric tissue specimens were diagnosed pathologically. AHR, AHRR, and $H$. pylori infection status in tissues were detected by immunohistochemistry. Human gastric cells were cocultured with $H$. pylori. siRNAs were used to silence AHR or AHRR, and a C57bl/6 mouse model colonized by $H$. pylori was established. Protein expression was determined by western blotting analysis, and TNF, IL-8 and IL-1 $\beta$ in cell supernatants were measured by ELISA.

Results: AHR and AHRR were expressed in gastritis tissues and gastric cancer tissues without $H$. pylori infection, and principally located in the cytoplasm and nucleus. AHR expression was significantly correlated with AHRR expression in gastric tissues without $H$. pylori infection $(P=0.008)$. However, their expressions were negatively correlated with $H$. pylori infection status. $H$. pylori coculture inhibited AHR and AHRR expression in stomach mucosa in vitro and in vivo. Gastric cells produced more TNF, IL-8 and IL- $1 \beta$ when AHR or AHRR was silenced.

Conclusions: This preliminary study indicates that AHR and AHRR may be involved in $H$. pylori-related gastric pathogenesis, and helps toward understanding of inflammation-initiated carcinogenesis of gastric cancer.
\end{abstract}

Key words: aryl hydrocarbon receptor, aryl hydrocarbon receptor repressor, H. pylori, gastric cancer

\section{Introduction}

Gastric cancer is one of the most common types of cancer, and rates as the second highest cancer-related cause of death worldwide [1, 2].
Persistent Helicobacter pylori colonization elicits chronic gastritis and aberrant epithelial cell proliferation, increasing the risk of gastric cancer 
development [3]. H. pylori secretes many pathogenic virulence factors that play important roles in gastric cancer pathogenesis [4]. Therefore, H. pylori has been identified as a major carcinogen for gastric adenocarcinoma [5].

Aryl hydrocarbon receptor (AHR) is a ligand-activated transcription factor belonging to the periodicity/aryl hydrocarbon receptor nuclear translocator/single-minded (PAS) superfamily of basic helix-loop-helix transcription factors [6]. Aryl hydrocarbon receptor repressor (AHRR) has been identified as an AHR-regulated gene [7]. Activation of the AHR signaling pathway leads to nuclear translocation of AHR, where it forms a heterodimer with AHR nuclear translocator [8]. The heterodimer binds to xenobiotic responsive elements located in enhancer regions of the target AHRR gene, and modulates AHRR transcription [9]. Recently, AHR was shown to sense distinct bacterial virulence factors and to control antibacterial responses [10]. In addition, exposure to bacterial lipopolysaccharide activates AHR and hepatic enzyme tryptophan 2,3-dioxygenase, an activating ligand for AHR, to downregulate inflammatory gene expression [11]. AHRR expression was shown to be downregulated in human malignant tissues from different anatomical origins, including the stomach, suggesting that AHRR is a putative tumor suppressor gene in multiple types of human cancers [12]. However, the role of AHR or AHRR in $H$. pylori-related gastric diseases remains unclear.

In the present study, we found that AHR and AHRR expression was reduced in gastritis tissues and gastric cancer tissues with $H$. pylori infection compared with $H$. pylori-negative tissues. We further found that $H$. pylori infection inhibited AHR and AHRR expression in stomach mucosa in vitro and in vivo, and that AHR or AHRR silencing promoted TNF, IL-8, and IL-1 $\beta$ secretion in human gastric mucosa cells cocultured with $H$. pylori. These preliminary results indicate that AHR and AHRR may be involved in $H$. pylori-related gastric pathogenesis.

\section{Methods}

\section{Human gastric tissue specimens}

Gastritis tissue specimens and gastric adenocarcinoma tissue specimens were obtained from patients undergoing endoscopic biopsy in the Third People's Hospital of Nantong, and patients diagnosed with gastric cancer undergoing radical gastrectomy in the First Affiliated Hospital of Nanjing Medical University, respectively. The patients were enrolled randomly, and none of the gastric cancer patients received preoperative chemotherapy or radiation therapy. All tissue specimens were diagnosed pathologically by two pathologists. Chronic gastritis was diagnosed according to the updated Sydney System [13], and gastric adenocarcinoma was diagnosed according to the American Joint Committee on Cancer criteria. All patients provided written informed consent. The study protocol was approved by the Institutional Review Board of the Third People's Hospital of Nantong and Institutional Review Board of Nanjing Medical University, and complied with the Helsinki Declaration.

\section{Cell culture}

Human gastric epithelial cell line GES-1 (CBTCCCAS, Shanghai, China) and gastric cancer cell lines AGS (ATCC, Manassas, VA) and SGC7901 (CBTCCCAS) were cultured in RPMI-1640 medium (Invitrogen, Carlsbad, CA) supplemented with 10\% fetal bovine serum (Hyclone, Logan, UT), penicillin/ streptomycin (1:100 dilution; Sigma-Aldrich, St. Louis, MO), and $4 \mathrm{mM}$ glutamine (Life Technologies, Gibco BRL, Grand Island, NY) at $37^{\circ} \mathrm{C}$ in a humidified atmosphere containing $5 \% \mathrm{CO}_{2}$.

\section{$H$. pylori culture and coculture with gastric cells}

Experiments were performed with the cytotoxic $\left(\mathrm{CagA}^{+} / \mathrm{VacA}^{+}\right)$reference strain of $H$. pylori 26695 (ATCC). H. pylori was grown under microaerophilic conditions on Columbia agar plates (bioMérieux, Marcy-l'Etoile, France) containing 100 U/ml H. pylori selective supplement (Oxoid, Basingstoke, UK) at $37^{\circ} \mathrm{C}$ in an anaerobic chamber (BBL Campy Pouch System; Becton Dickinson Microbiology Systems, San Diego, CA) for 48-72 h. The cells were harvested and resuspended in antibiotic-free RPMI-1640 medium (Invitrogen) supplemented with $2 \%$ fetal calf serum (Sigma-Aldrich). The bacterial densities were adjusted by optical density (OD) measurements at $660 \mathrm{~nm}$, in which $1 \mathrm{OD}_{660}=1 \times 10^{8}$ colony-forming units $(\mathrm{CFU}) / \mathrm{ml}$. H. pylori was then incubated with GES-1, AGS, or SGC7901 cells at a bacteria/cell ratio of 100:1 in culture medium for the required times.

\section{Western blotting analysis}

Western blotting analyses were performed to detect protein expression as described previously [14]. The following antibodies were used: polyclonal rabbit anti-AHR antibody (Santa Cruz Biotechnology, Santa Cruz, CA); mouse monoclonal anti-AHRR antibody (Absci, Baltimore, MD); and anti- $\beta$-actin antibody (Cell Signaling Technology, Beverly, MA). Protein expression was quantified by densitometric analysis, and the target protein expression levels were normalized by the $\beta$-actin expression level. 
Immunohistochemical detection of AHR, AHRR, and $H$. pylori infection status in tissues

AHR, AHRR, and H. pylori infection status in gastric tissues specimens were detected by immunohistochemistry (IHC) as described previously [15, 16, 17]. A polyclonal rabbit anti-AHR antibody (Santa Cruz Biotechnology), mouse monoclonal anti-AHRR antibody (Abcam, Cambridge, UK), and monoclonal mouse anti-human $H$. pylori antibody (Gene Tech, Shanghai, China) were used. IHC was performed on paraffin-embedded formalin-fixed tissues according to standard protocols. The IHC results for AHR or AHRR expression were scored by the percentages of stained cells as follows:,$- 0 \% ;+, 1-25 \% ;++, 25-50 \%$; ,$+++>50 \%$. H. pylori infection status was determined as positive or negative.

\section{Establishment of a C57bl/6 mouse model colonized by $\boldsymbol{H}$. pylori}

Thirty 4-week-old female $\mathrm{C} 57 \mathrm{bl} / 6$ mice (Vitalriver, Nanjing, China) weighing 20-22 g were randomly divided into an $H$. pylori treatment group $(n=15)$ and a control group $(n=15)$. Mice in the $H$. pylori treatment group were orally inoculated with 0.3 $\mathrm{ml}$ of phosphate-buffered saline (PBS) containing $H$. pylori 26695 suspended at $3 \times 10^{7} \mathrm{CFU} / \mathrm{ml}$ using a feeding needle. H. pylori treatment was performed for 3 days during 8 consecutive weeks. Before inoculation, the mice were fasted for $12 \mathrm{~h}$ and slowly pretreated with $0.2 \mathrm{ml}$ of oral $5 \% \mathrm{NaHCO}_{3}$ at $30 \mathrm{~min}$ before inoculation. Mice in the control group were inoculated with PBS only. At 8 weeks after inoculation, gastric mucous tissues in the antrum were collected for analyses.

\section{Detection of TNF, IL-8, and IL- $1 \beta$ in cell supernatants by ELISA}

H. pylori was incubated with GES-1, AGS, and SGC7901 cells for up to $24 \mathrm{~h}$ in culture medium. The bacteria/cell ratio was 100:1. The cells were then washed twice with PBS and cultured in serum-free RPMI-1640 medium. After $24 \mathrm{~h}$, the culture supernatants were collected for protein detection. Secretion of TNF, IL-8, and IL-1 $\beta$ was detected by ELISA (SenBeiJia Biological Technology, Nanjing, China) according to the manufacturer's protocol. In each group, the amounts of TNF, IL- 8 , and IL- $1 \beta$ were measured spectrophotometrically by the absorbance at $450 \mathrm{~nm}$. Each experiment was repeated at least three times.

\section{Transduction of AHR and AHRR siRNAs}

AHR and AHRR siRNAs were synthesized by Ribobio (Guangzhou, China). Transduction of AHR-siRNA or AHRR-siRNA was performed using a
riboFect $^{\mathrm{TM}}$ CP Transfection Kit (Ribobio, Guangzhou, China) according to the manufacturer's protocol. Expression of AHR or AHRR was determined by western blotting analyses.

\section{Statistical analysis}

Data are expressed as mean \pm standard error. In experiments involving protein expression, the data were representative of three independent experiments. Pearson's chi-square test was used to examine associations between protein expression levels and various clinicopathological parameters. Quantitative data were compared between the control and treatment groups by analysis of variance. All analyses were performed with SPSS software (version 19.0; SPSS Inc., Chicago, IL). Values of $P<0.05$ were considered to indicate statistical significance.

\section{Results}

AHR or AHRR expression in gastritis tissues and gastric cancer tissues is correlated with $H$. pylori infection status

To investigate the role of AHR or AHRR in $H$. pylori-related gastric diseases, we detected AHR and AHRR expression in gastritis tissues and gastric cancer tissues. As shown in Figure 1 and Figure 2, AHR and AHRR were expressed in gastritis tissues and gastric cancer tissues without $H$. pylori infection, and principally located in the cytoplasm and nucleus. However, AHR and AHRR were scarcely detected in gastric tissues with $H$. pylori infection.

We evaluated the correlation between AHR or AHRR expression in gastric tissues and $H$. pylori infection status. As shown in Table $\mathbf{1}$ and Table 2, AHR or AHRR expression was significantly associated with non- $H$. pylori infection status in gastritis tissues and gastric cancer tissues, i.e. AHR or AHRR expression was negatively correlated with $H$. pylori infection status. Furthermore, AHR expression was significantly correlated with AHRR expression in gastric tissues without $H$. pylori infection $(P=0.008)$. These results indicate that $H$. pylori infection may lead to downregulation of AHR and AHRR.

\section{$H$. pylori infection inhibits AHR and AHRR expression in stomach mucosa in vitro and in vivo}

To examine the effects of $H$. pylori infection on AHR or AHRR expression in the stomach mucosa, human gastric mucosa GES-1, AGS, and SGC7901 cells were cocultured with $H$. pylori strain 26695 at a bacteria/cell ratio of 100:1. As shown in Figure 3, $H$. pylori infection inhibited AHR and AHRR expression in these cell lines in an time-dependent manner. 
Furthermore, a C57bl/ 6 mouse model colonized by $H$. pylori was established. IHC analyses revealed many bacteria colonizing the stomach in the H. pylori infection group (Figure 4B), while no bacteria were found in the control group (Figure 4A). In the control group, AHR and AHRR were expressed in the gastric mucosa (Figure 4C and 4E). In contrast, AHR and AHRR expression in the stomach was significantly reduced or eliminated in the $H$. pylori infection group (Figure 4D and 4F), demonstrating that AHR and AHRR may be involved in the pathogenesis of $H$. pylori infection.

\section{AHR or AHRR silencing promotes TNF, IL-8, and IL- $1 \beta$ secretion in human gastric mucosa cells cocultured with $\boldsymbol{H}$. pylori}

Secretion of multiple proinflammatory cytokines, including TNF, IL-8, and IL- $1 \beta$, has been identified as a hallmark of gastric mucosa infected by H. pylori, and these cytokines play significant roles in the development of stomach mucosal diseases associated with $H$. pylori infection [18]. To examine the role of AHR or AHRR in the pathogenesis of H. pylori infection, gastric mucosa GES-1, AGS and SGC7901 cells were transfected with AHR-siRNA or AHRR-siRNA (Figure 5 and Figure 6). AHRR expression was simultaneously inhibited in cells when AHR was silenced (Figure 5A), and AHR was down-regulated when AHRR was silenced (Figure 6A). Secretion of TNF, IL- 8 , and IL-1 $\beta$ by the transfected cells was measured by ELISA in cell supernatants after coculture with $H$. pylori for $24 \mathrm{~h}$. The cells produced more proinflammatory cytokines when AHR or AHRR was silenced (Figure 5B and Figure 6B). These findings further support the involvement of AHR and AHRR in the pathogenesis of $H$. pylori infection.

Table 1. AHR, AHRR, and $H$. pylori infection status in gastritis tissues

\begin{tabular}{llllll}
\hline Variables & $\begin{array}{l}\text { Expression } \\
\text { intensity }\end{array}$ & \multicolumn{2}{c}{ H. pylori infection } & \multirow{2}{*}{$\chi^{2}$} & P value \\
\cline { 2 - 4 } & $-/+$ & $6(33.33)$ & $17(73.91)$ & 6.75 & 0.013 \\
\hline AHR expression & $-/+$ & $12(66.67)$ & $6(26.09)$ & & \\
[n (\%)] & $++\sim+++$ & $5(27.78)$ & $15(65.22)$ & 5.67 & 0.028 \\
AHRR expression & $-/+$ & $13(72.22)$ & $8(34.78)$ & & \\
[n (\%)] & $++\sim+++$ & &
\end{tabular}

Table 2. AHR, AHRR, and H. pylori infection status in gastric cancer tissues

\begin{tabular}{llllll}
\hline Variables & $\begin{array}{l}\text { Expression } \\
\text { intensity }\end{array}$ & \multicolumn{2}{l}{ H. pylori infection } & \multirow{2}{*}{$\chi^{2}$} & \multirow{2}{*}{$\boldsymbol{P}$ value } \\
\cline { 3 - 4 } & & - & + & & \\
\hline AHR expression & $-/+$ & $6(30.00)$ & $15(68.18)$ & 6.11 & 0.029 \\
[n (\%)] & $++\sim+++$ & $14(70.00)$ & $7(31.82)$ & & \\
AHRR expression & $-/+$ & $5(25.00)$ & $17(77.27)$ & 11.48 & 0.005 \\
[n (\%)] & $++\sim+++$ & $15(75.00)$ & $5(22.73)$ & & \\
\hline
\end{tabular}

\section{AHR}

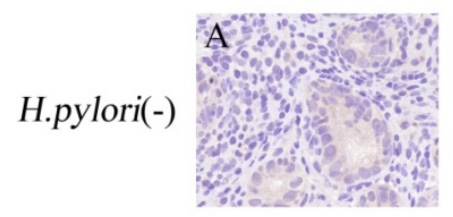

H.pylori(+)
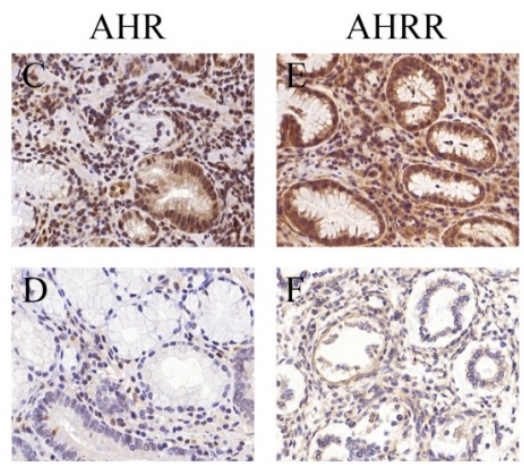

Figure 1. AHR or AHRR expression is correlated with $H$. pylori infection status in gastritis tissues. (A and $\mathrm{B}$ ) $H$. pylori infection status was detected by IHC in gastric mucosa, and observed as yellow or brown staining in the lumen of gastric glands (B). (C-F) AHR (C) or AHRR (E) was expressed in gastric mucosa without $H$. pylori infection, but neither AHR (D) nor AHRR (F) was expressed in tissues with $H$. pylori infection. Original magnification, $\times 200$.

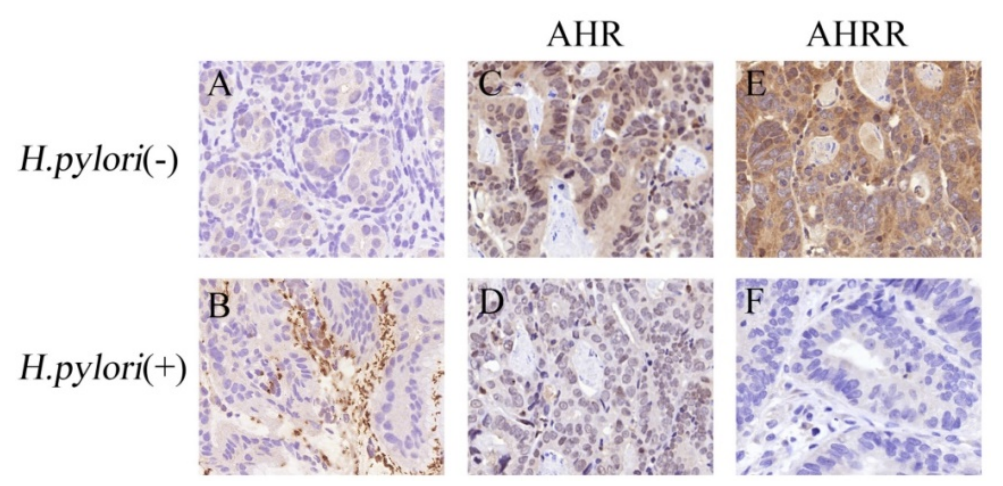

Figure 2. AHR or AHRR expression is correlated with $H$. pylori infection status in gastric cancer tissues. (A and $B) H$. pylori infection status was detected by lHC in gastric mucosa, and observed as yellow or brown staining in the lumen of gastric glands (B). (C-F) AHR (C, 2+) or AHRR (E, 3+) was expressed in gastric mucosa without $H$. pylori infection, but neither AHR (D) nor AHRR (F) was expressed in tissues with H. pylori infection. Original magnification, $\times 200$. 

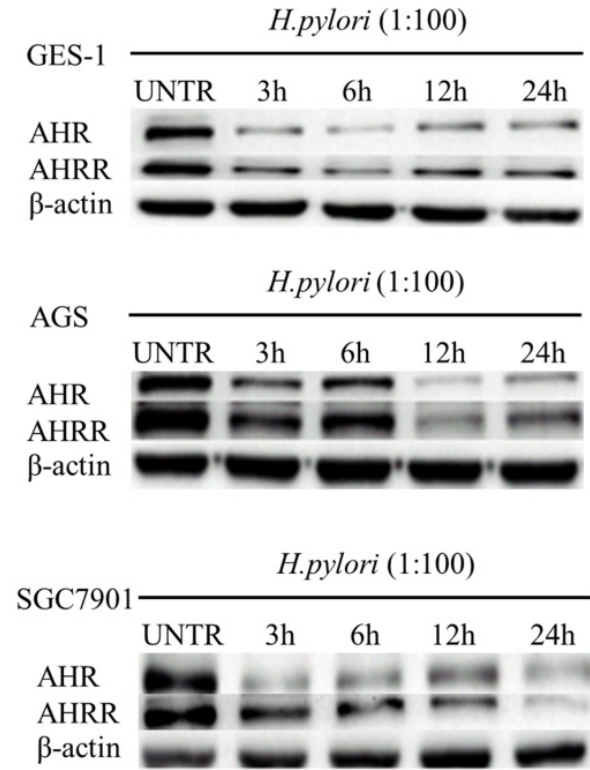

Figure 3. $H$. pylori infection inhibits AHR or AHRR expression in gastric cell lines. Human gastric mucosa cell line GES-1, AGS, and SGC7901 cells were cocultured with $H$. pylori strain 26695 at a bacteria/cell ratio of 100:1. Expression of AHR and AHRR in these cell lines was inhibited in a time-dependent manner.

\section{Discussion}

H. pylori has been identified as an important risk factor for gastritis, gastric ulcer, gastric mucosa-associated lymphoid tissue lymphoma, and gastric cancer [19-21]. About 90\% of non-cardiac adenocarcinoma was reported to be associated with H. pylori infection [22]. Accumulating studies have demonstrated that eradication of $H$. pylori infection reduced the risk of gastric cancer and peptic ulcer [23, 24], risk of gastric cancer-related death [25], and incidence of second primary gastric cancer [26]. However, the pathogenesis of $H$. pylori infection in gastric diseases remains uncertain. Tan et al. [27] described that $H$. pylori infection was involved in the transformation process of normal gastric mucosa, intestinal metaplasia, atypical hyperplasia, and early gastric cancer lesions. Wang et al. [28] reported that $H$. pylori infection upregulated aquaporin 3 (AQP3) expression in human gastric cancer cells and promoted gastric cancer cell migration.

The roles of AHR and AHRR in gastric diseases remain controversial. Wei et al. [29] showed that benzopyrene promoted gastric cancer cells to undergo metastasis through the AHR and ERK signaling pathway. However, AHR expression was demonstrated to gradually increase from gastritis to gastric cancer [15], and a selective AHR modulator, 3,3'-diindolylmethane, suppressed gastric cancer cell growth and peritoneal metastasis [30]. A novel AHR inhibitor, biseugenol, inhibited peritoneal dissemination of gastric tumors by inhibiting epithelial-tomesenchymal transition via AHR [31]. Li et al. [16] demonstrated that decreased expression of AHRR was significantly associated with poor prognosis in gastric adenocarcinoma patients.

In this study, we showed that AHR and AHRR were expressed in gastritis tissues and gastric cancer tissues, and that this expression was negatively associated with $H$. pylori infection status. Therefore, $H$. pylori infection may inhibit AHR and AHRR expression in the stomach, and the in vitro and in vivo results supported this speculation. H. pylori infection stimulated stomach cells to produce proinflammatory factors [32, 33], and these cytokines exerted provital roles in the pathogenesis of $H$. pylori infectionassociated gastric diseases, including gastritis and gastric cancer [34, 35, 36, 37]. The current study demonstrated that silencing of AHR or AHRR promoted TNF, IL-8 and IL-1 $\beta$ secretion in human stomach cells after coculture with $H$. pylori. We reported previously that $H$. pylori infection increases the mRNA expression of IL-6, IL-8, and TNF in gastric

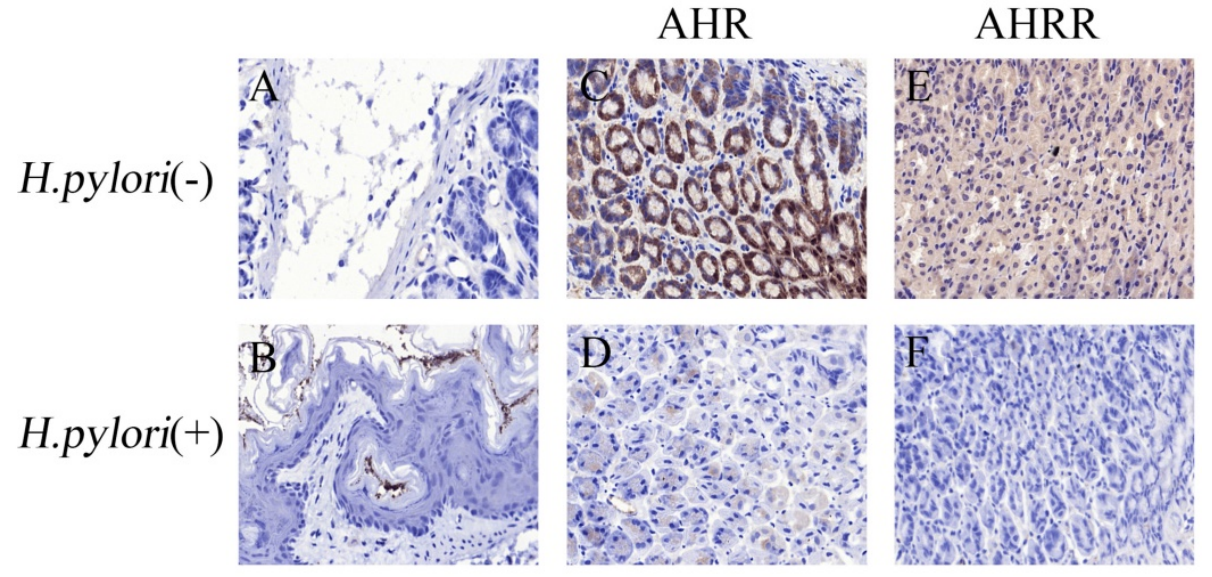

Figure 4. $H$. pylori infection inhibits AHR or AHRR expression in the stomach. (A-F) AHR, AHRR, and $H$. pylori infection status were detected by IHC in a mouse model. In the $H$. pylori-negative control group (A), AHR (C) or AHRR (E) was expressed. In the H. pylori-infected group (B), neither AHR (D) nor AHRR (F) was detected. Original magnification $\times 100$. 
mucosa with regarding to that in the control groups in mice [38]. These results provide direct evidence that AHR and AHRR may be involved in the pathogenesis of $H$. pylori infection.

There is no current evidence on how $H$. pylori infection controls AHR or AHRR expression in stomach. The associations between gut microbiomeAHR crosstalk have been established [39]. Several microbial metabolites, such as lactobacilli spp metabolites, can enhance the mucosal resistance against the inflammation via activation of AHR [40], and dietary 2,3,7,8-tetrachlorodibenzofuran can alter the gut microbiota via AHR activation [41], which suggests that AHR may not only act as a ligandactivating transcription factor, but may also interact with microbiome to regulate barrier function of gastric mucosa. It may be hypothesized that normal AHR or AHRR function is needed to resist H. pylori colonization and to defense against the inflammation.
A
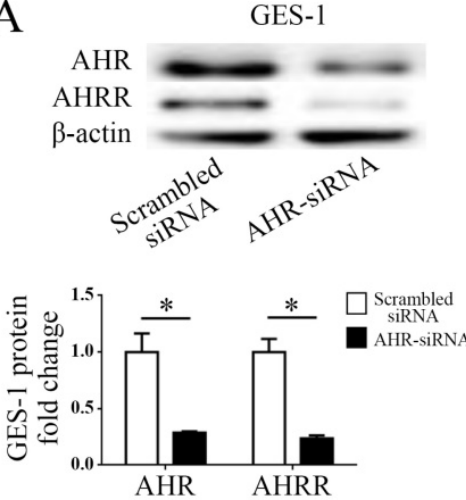

B

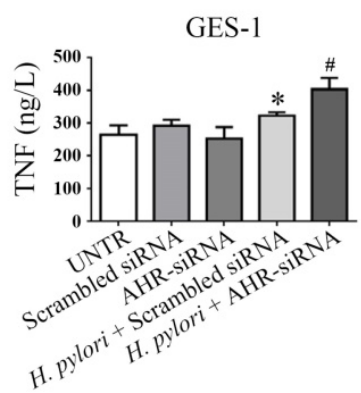

GES-1
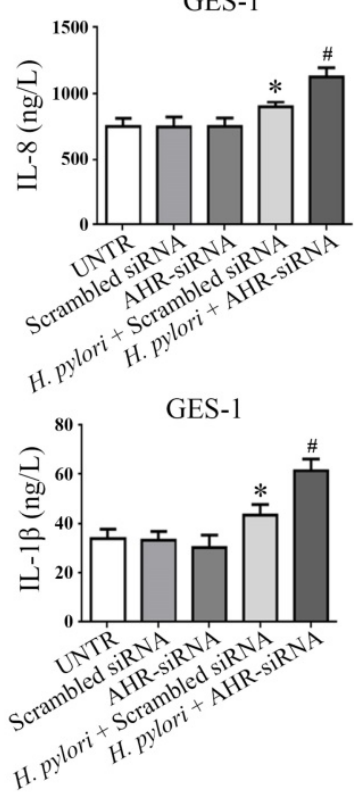

AGS
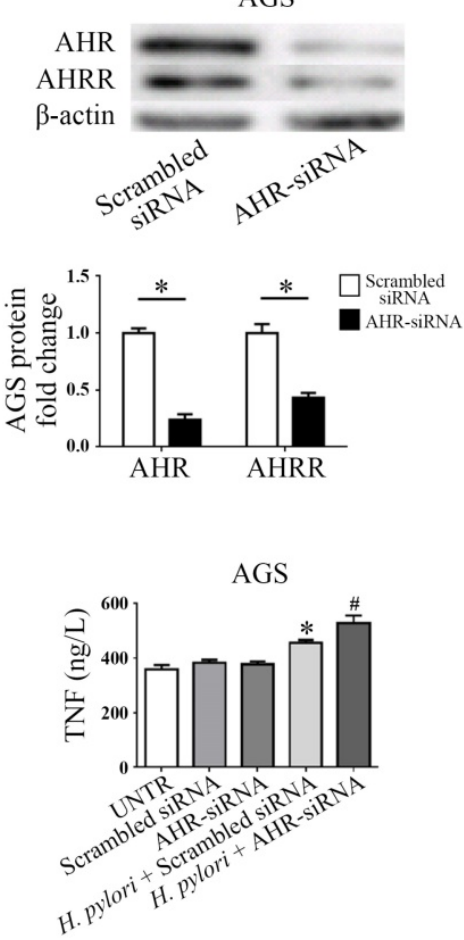

AGS
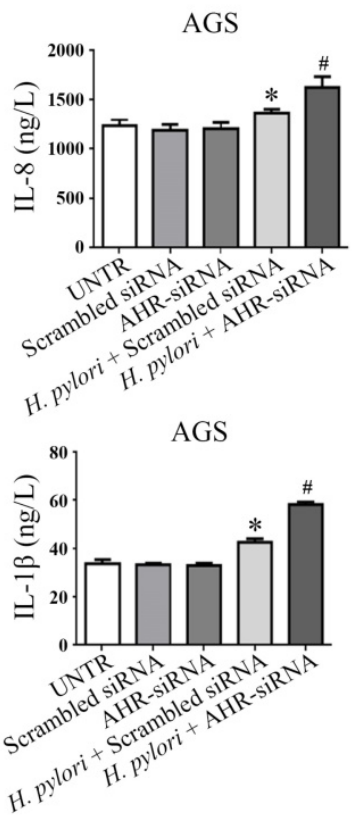

SGC7901
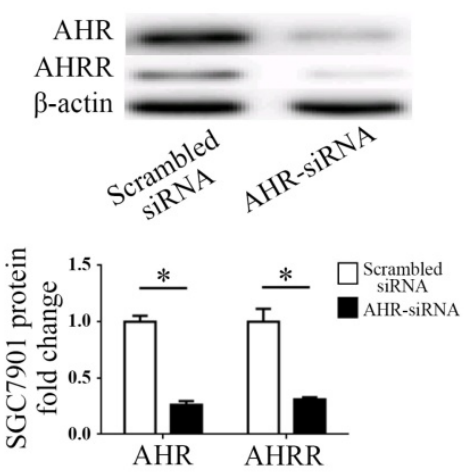

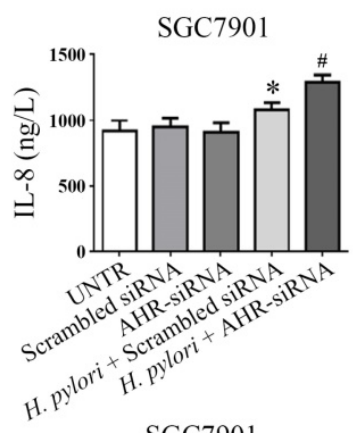

SGC7901

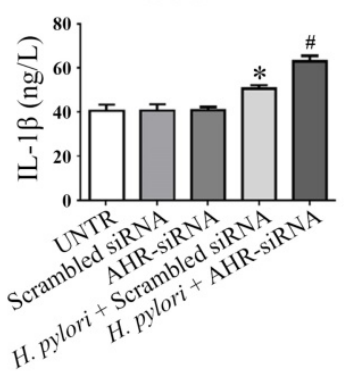

Figure 5. AHR silencing inhibits AHRR expression simultaneously, and increases secretion of TNF, IL-8, and IL-1 $\beta$. (A) AHRR expression was inhibited in GES-1, AGS, and SGC7901 cells when AHR was silenced $(* P<0.05)$. (B) Secretion of TNF, IL-8, and IL-1 $\beta$ by the cells was measured by ELISA in cell supernatants after coculture with $H$. pylori for $24 \mathrm{~h}$. The cells produced more TNF, IL-8, and IL-1 $\beta$ when AHR was silenced $(* P<0.05$ vs. UNTR group, scrambled siRNA group, and AHR-siRNA group; $\# P<0.05$ vs. AHR-siRNA group). UNTR: untreated group. 
A
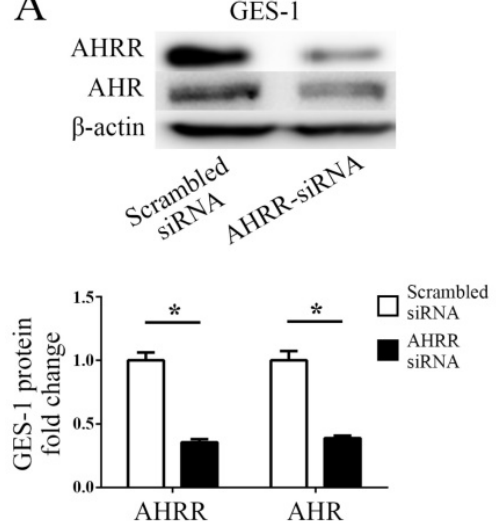

$\mathrm{B}$

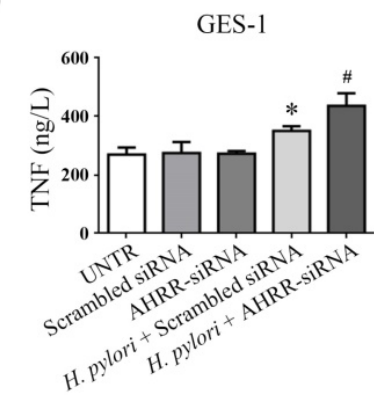

GES-1

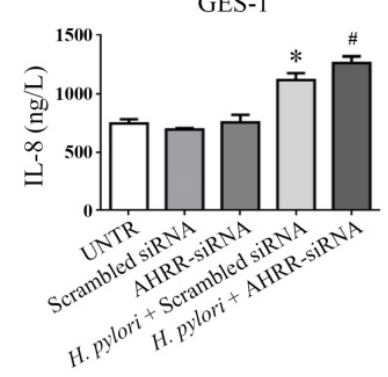

GES-1

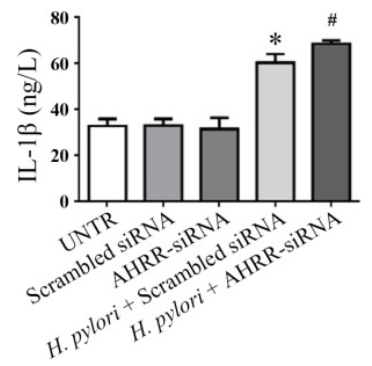

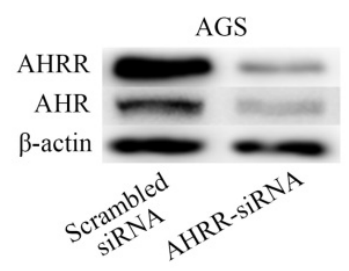

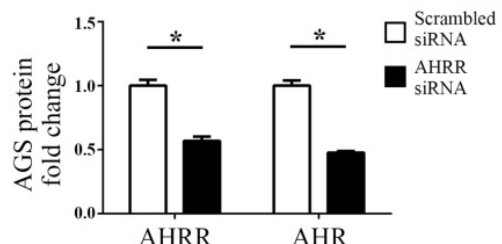

AGS

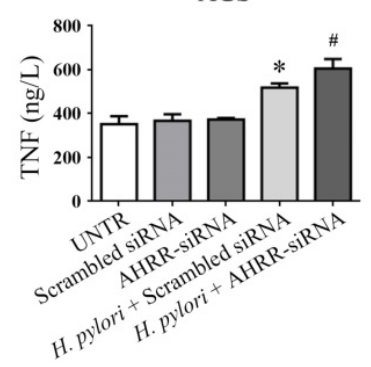

AGS

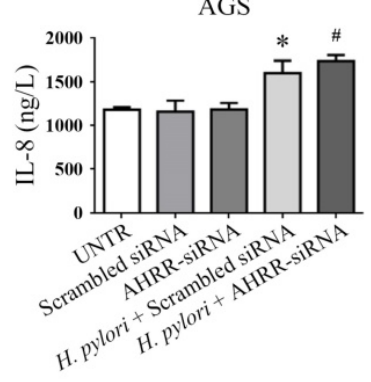

AGS

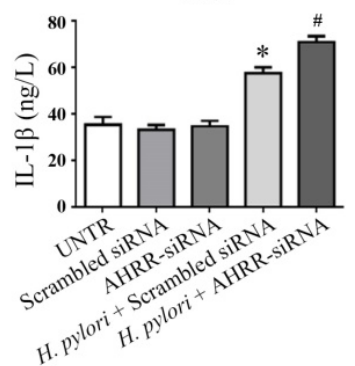

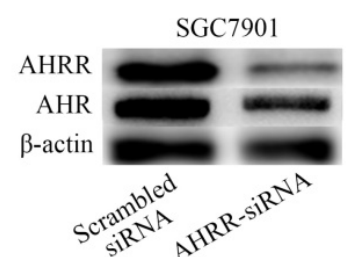
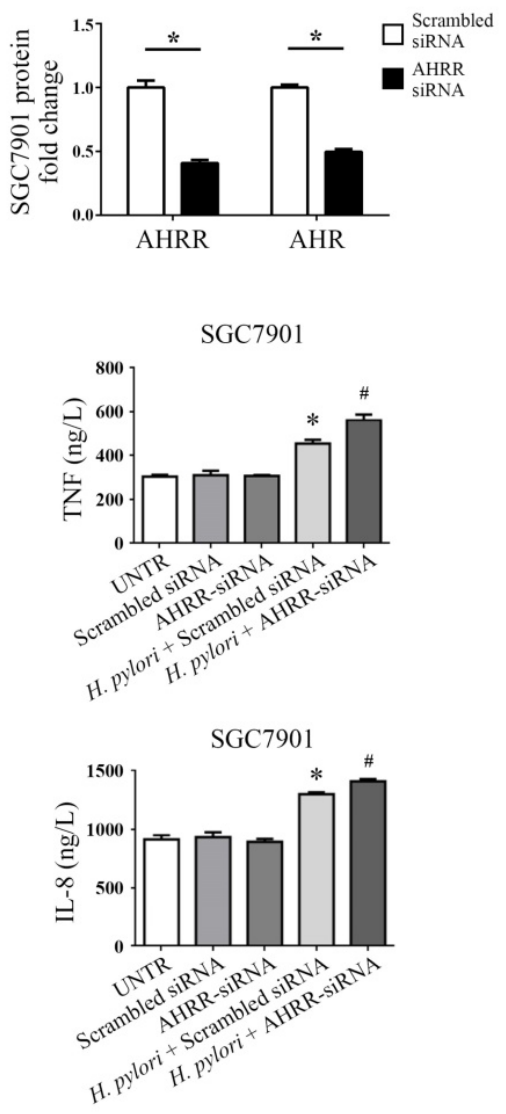

SGC7901

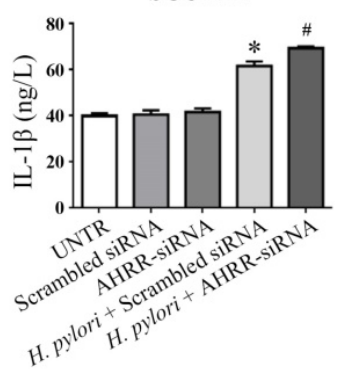

Figure 6. AHRR silencing upregulates secretion of TNF, IL-8, and IL-1 1 . (A) AHRR expression was inhibited by AHRR silencing, and AHR was down-regulated simultaneously $(* P<0.05)$. (B) AHRR-silenced GES-1, AGS, and SGC7901 cells produced more TNF, IL-8, and IL-1 $\beta$ after coculture with $H$. pylori for $24 \mathrm{~h}(* P<0.05$ vs. UNTR group, scrambled siRNA group, and AHRR-siRNA group; $\# P<0.05$ vs. AHRR-siRNA group). UNTR: untreated group.

In summary, this preliminary study provides novel insights into the mechanism of H. pylori infection in the stomach, and helps toward understanding of inflammation-initiated carcinogenesis of gastric cancer. Further experiments are certainly required to reveal the more precise mechanisms underlying AHR or AHRR regulation by H. pylori infection in the stomach.

\section{Acknowledgments}

This work was funded by the National Natural Science Foundation of China (Grant No. 81272711 to L. Shen), the Priority Academic Program Development of Jiangsu Higher Education Institutions (PAPD, JX10231801 to L. Shen), the Key Medical Talents Program of Jiangsu Province (ZDRCA2016014 to L. Shen), and the Key R \& D Program of Jiangsu Province (Social Development, BE2018758 to L. Shen). 


\section{Competing Interests}

The authors have declared that no competing interest exists.

\section{References}

1. Chen W, Zheng R, Baade PD, et al. Cancer statistics in China, 2015. CA Cancer J Clin. 2016; 66: 115-32.

2. Hamashima C. Current issues and future perspectives of gastric cancer screening. World J Gastroenterol. 2014; 20: 13767-74.

3. Kiga K, Mimuro H, Suzuki M, et al. Epigenetic silencing of miR-210 increases the proliferation of gastric epithelium during chronic Helicobacter pylori infection. Nat Commun. 2014; 5: 4497.

4. Barrozo RM, Hansen LM, Lam AM, et al. CagY is an immune-sensitive regulator of the Helicobacter pylori type IV secretion system. Gastroenterology. 2016; 151:1164-75.

5. Peleteiro B, Bastos A, Ferro A, et al. Prevalence of Helicobacter pylori infection worldwide: a systematic review of studies with national coverage. Dig Dis Sci. 2014; 59:1698-709.

6. Kewley RI, Whitelaw ML, Chapman-Smith A. The mammalian basic helix-loop-helix/PAS family of transcriptional regulators. Int J Biochem Cell Biol. 2004; 36:189-204.

7. Mimura J, Ema M, Sogawa K, et al. Identification of a novel mechanism of regulation of $\mathrm{Ah}$ (dioxin) receptor function. Genes Dev. 1999; 13: 20-5.

8. Reisz-Porszasz S, Probst MR, Fukunaga BN, et al. Identification of functional domains of the aryl hydrocarbon receptor nuclear translocator protein (ARNT). Mol Cell Biol. 1994; 14: 6075-86.

9. Haarmann-Stemmann $\mathrm{T}$, Bothe $\mathrm{H}$, Kohli A, et al. Analysis of the transcriptional regulation and molecular function of the aryl hydrocarbon receptor repressor in human cell lines. Drug Metab Dispos. 2007; 35: 2262-9.

10. Moura-Alves $\mathrm{P}, \mathrm{Fae} \mathrm{K}$, Houthuys E, et al. AhR sensing of bacterial pigments regulates antibacterial defence. Nature. 2014; 512: 387-92.

11. Bessede A, Gargaro M, Pallotta MT, et al. Aryl hydrocarbon receptor control of a disease tolerance defence pathway. Nature. 2014; 511: 184-90.

12. Zudaire E, Cuesta N, Murty V, et al. The aryl hydrocarbon receptor repressor is a putative tumor suppressor gene in multiple human cancers. J Clin Invest. 2008; 118: 640-50.

13. Dixon MF, Genta RM, Yardley JH, et al. Classification and grading of gastritis. The updated Sydney System. International Workshop on the Histopathology of Gastritis, Houston 1994. Am J Surg Pathol. 1996; 20: 1161-81.

14. Wang J, Gui Z, Deng L, et al. c-Met upregulates aquaporin 3 expression in human gastric carcinoma cells via the ERK signalling pathway. Cancer Lett. 2012; 319: 109-17.

15. Peng TL, Chen J, Mao W, et al. Potential therapeutic significance of increased expression of aryl hydrocarbon receptor in human gastric cancer. World J Gastroenterol. 2009; 15: 1719-29.

16. Li YF, Wang DD, Zhao BW, et al. Poor prognosis of gastric adenocarcinoma with decreased expression of AHRR. PLoS One. 2012; 7: e43555.

17. He Q, Li G, Wang X, et al. A decrease of histone deacetylase 6 expression caused by Helicobacter pylori infection is associated with oncogenic transformation in gastric cancer. Cell Physiol Biochem. 2017; 42: 1326-35.

18. Alzahrani S, Lina TT, Gonzalez J, et al. Effect of Helicobacter pylori on gastric epithelial cells. World J Gastroenterol. 2014; 20: 12767-80.

19. Lanas A, Chan F. Peptic ulcer disease. Lancet. 2017; 390: 613-24.

20. Fock KM, Graham DY, Malfertheiner P. Helicobacter pylori research: historical insights and future directions. Nat Rev Gastroenterol Hepatol. 2013; 10: 495-500.

21. Malfertheiner P, Megraud F, O'Morain CA, et al. Management of Helicobacter pylori infection--the Maastricht IV/Florence Consensus Report. Gut. 2012; 61: 646-64.

22. de Martel C, Ferlay J, Franceschi S, et al. Global burden of cancers attributable to infections in 2008: a review and synthetic analysis. Lancet Oncol. 2012; 13: 607-15.

23. Fuccio L, Zagari RM, Eusebi LH, et al. Meta-analysis: can Helicobacter pylori eradication treatment reduce the risk for gastric cancer? Ann Intern Med. 2009; 151: $121-8$.

24. Venerito M, Nardone G, Selgrad M, et al. Gastric cancer-epidemiologic and clinical aspects. Helicobacter. 2014; 19 (Suppl 1): 32-7.

25. Ford AC, Forman D, Hunt RH, et al. Helicobacter pylori eradication therapy to prevent gastric cancer in healthy asymptomatic infected individuals: systematic review and meta-analysis of randomised controlled trials. BMJ. 2014; 348: g3174

26. Chen HN, Wang Z, Li X, et al. Helicobacter pylori eradication cannot reduce the risk of gastric cancer in patients with intestinal metaplasia and dysplasia: evidence from a meta-analysis. Gastric Cancer. 2016; 19: 166-75.

27. Wang G, Gao F, Zhang W, et al. Involvement of Aquaporin 3 in Helicobacter pylori-related gastric diseases. PLoS One. 2012; 7: e49104.

28. Tan P, Yeoh KG. Genetics and molecular pathogenesis of gastric adenocarcinoma. Gastroenterology. 2015; 149: 1153-62.

29. Wei $\mathrm{Y}$, Zhao L, He W, et al. Benzo[a]pyrene promotes gastric cancer cell proliferation and metastasis likely through the Aryl hydrocarbon receptor and ERK-dependent induction of MMP9 and c-myc. Int J Oncol. 2016; 49: 2055-63.
30. Yin XF, Chen J, Mao W, et al. A selective aryl hydrocarbon receptor modulator 3,3'-Diindolylmethane inhibits gastric cancer cell growth. J Exp Clin Cancer Res. 2012; 31: 46.

31. Lai DW, Liu SH, Karlsson AI, et al. The novel Aryl hydrocarbon receptor inhibitor biseugenol inhibits gastric tumor growth and peritoneal dissemination. Oncotarget. 2014; 5: 7788-804.

32. Lin $\mathrm{Q}, \mathrm{Xu} \mathrm{H}, \mathrm{Chen} \mathrm{X}$, et al. Helicobacter pylori cytotoxin-associated gene $\mathrm{A}$ activates tumor necrosis factor-alpha and interleukin-6 in gastric epithelial cells through P300/CBP-associated factor-mediated nuclear factor-kappaB p65 acetylation. Mol Med Rep. 2015; 12: 6337-45.

33. Zou M, Wang $\mathrm{F}$, Jiang A, et al. MicroRNA-3178 ameliorates inflammation and gastric carcinogenesis promoted by Helicobacter pylori new toxin, Tip-alpha, by targeting TRAF3. Helicobacter. 2017; [Epub ahead of print].

34. Rossi $\mathrm{AF}$, Cadamuro $\mathrm{AC}$, Biselli-Perico $\mathrm{JM}$, et al. Interaction between inflammatory mediators and miRNAs in Helicobacter pylori infection. Cell Microbiol. 2016; 18: 1444-58.

35. Inoue $\mathrm{K}$, Shiota $\mathrm{S}$, Yamada $\mathrm{K}$, et al. Evaluation of a new tumor necrosis factor-alpha-inducing membrane protein of Helicobacter pylori as a prophylactic vaccine antigen. Helicobacter. 2009; 14: 135-43.

36. El-Omar EM, Rabkin CS, Gammon MD, et al. Increased risk of noncardia gastric cancer associated with proinflammatory cytokine gene polymorphisms. Gastroenterology. 2003; 124: 1193-201.

37. Figueiredo C, Machado JC, Pharoah P, et al. Helicobacter pylori and interleukin 1 genotyping: an opportunity to identify high-risk individuals for gastric carcinoma. J Natl Cancer Inst. 2002; 94: 1680-7.

38. Wen J, Wang Y, Gao C, et al. Helicobacter pylori infection promotes Aquaporin 3 expression via the ROS-HIF-1a-AOP3-ROS loop in stomach mucosa: a potential novel mechanism for cancer pathogenesis. Oncogene. 2018; [Epub ahead of print].

39. Korecka A, Dona A, Lahiri S, et al. Bidirectional communication between the Aryl hydrocarbon receptor (AhR) and the microbiome tunes host metabolism. NPJ Biofilms Microbiomes. 2016; 2: 16014

40. Zelante T, Iannitti RG, Cunha C, et al. Tryptophan catabolites from microbiota engage aryl hydrocarbon receptor and balance mucosal reactivity via interleukin-22. Immunity. 2013; 39: 372-85.

41. Zhang L, Nichols RG, Correll J, et al. Persistent organic pollutants modify gut microbiota-host metabolic homeostasis in mice through Aryl hydrocarbon receptor activation. Environ Health Perspect. 2015; 123: 679-88. 